\title{
Prospects for Higgs boson measurements at the LHC in the $H \rightarrow \tau \tau$ decay mode
}

\section{Chris Boddy}

University of Oxford

E-mail: christopher.boddy@cern.ch

\section{Sinead Farrington}

University of Oxford

E-mail: s.farrington1@physics.ox.ac.uk

\section{Chris Hays}

University of Oxford

E-mail: hays@physics.ox.ac.uk

\begin{abstract}
The associated Higgs boson production modes $W H, Z H$, and $t \bar{t} H$, where the Higgs boson subsequently decays to a pair of tau leptons, can be used to measure the relative branching ratios of a low-mass Higgs boson at the Large Hadron Collider and hence measure the ratio of Yukawa couplings as predicted by the Standard Model. We find that these modes can enhance coupling-ratio sensitivity with $50 \mathrm{fb}^{-1}$ of $p p$ collision data at a center of mass energy equal to $14 \mathrm{TeV}$, for a Higgs boson mass between 115 and $135 \mathrm{GeV} / c^{2}$.
\end{abstract}

35th International Conference of High Energy Physics - ICHEP2010,

July 22-28, 2010

Paris France 


\section{Introduction}

The early collisions at the Large Hadron Collider (LHC) [1] are an important step on the path to discovery of the last unobserved particle of the Standard Model (SM), the Higgs boson. While the prospects for Higgs boson discovery and measurement have been studied extensively [2], lowrate processes observable with the full LHC design luminosity have not been completely explored. We investigate the sensitivity of $50 \mathrm{fb}^{-1}$ of LHC data to the Higgs boson production processes $W H$, $Z H$, and $t \bar{t} H$, followed by $H \rightarrow \tau \tau$ and at least one $W \rightarrow l v$ or $Z \rightarrow l l$ decay.

Combining these channels with the recently enhanced prospects for $W / Z / t \bar{t}+H \rightarrow b b$ observation [3] improves the expected LHC sensitivity to several quantities, most notably the Yukawa coupling ratio $g_{H b b} / g_{H \tau \tau}$, determined at tree level by the bottom-quark and tau-lepton masses, and sensitive to differences in the source of mass for quarks and leptons.

\section{Detector Simulation and Selection Criteria}

Associated Higgs boson production signal and background processes (including di-boson, vector boson + jet(s), $t \bar{t}$ and $t \bar{t}+$ jet(s) production) were generated using the Sherpa [5] and Herwig++ [6] Monte Carlo generators. Detector effects and acceptances were simulated using the Delphes framework [7], a fast simulation program that can be parameterised to approximate a detector with properties similar to the ATLAS and CMS detectors. Lepton and hadronically decaying tau $\left(\tau_{j e t}\right)$ identification and misidentifiaction rates [8] are summarised in Table 1. An example of the reconstructed object selection criteria is shown in Table 2 below for $W H$ events, with two leptons and one $\tau_{j e t}$ (all momenta are in $\mathrm{GeV} / c$ and masses are in $\mathrm{GeV} / c^{2}$ ).

\begin{tabular}{|c|c|c|c|}
\hline Object & ID efficiency (\%) & Jet mis-identification rate (\%) & Trigger efficiency (\%) \\
\hline$e$ & 64.2 & 0.0108 & 65 \\
$\mu$ & 94.2 & 0.169 & 90 \\
$\tau_{j e t}$ & 40 & 2.3 & lepton triggers used \\
\hline
\end{tabular}

Table 1: Simulated detector indentification and triggering efficiencies.

\begin{tabular}{|c|c|c|c|c|c|c|}
\hline$P_{T}^{l_{1}}$ & $P_{T}^{l_{2}}$ & $P_{T}^{\tau_{j e t}}$ & $\not_{T}$ & $m_{l^{+} l^{-}}$ & $\left|\sum_{\text {charges }}\right|$ & $\sum \overrightarrow{P_{T}}$ \\
\hline 25 & 15 & 25 & $20<\not_{T}<100$ & $<76$ or $>106$ & 1 & $<60$ \\
\hline
\end{tabular}

Table 2: $W H$ search channel selection criteria

\section{Monte Carlo Pseudo-experiment Results.}

Since momentum is carried away by at least two neutrinos in these events, conventional mass reconstruction is not possible. The invariant mass of the visible tau decay products is used to create templates in the $t \bar{t} H$ and $W H$ analyses while the collinear mass approximation [8] is used in the $Z H$ analysis to reconstruct the $\tau \tau$ mass.

Pseudo-experiments are generated from signal and background MC distributions according to a Poisson distribution, $P(k ; \lambda)=\frac{\lambda^{k} \exp ^{-\lambda}}{k !}$, for $k$ observed events and $\lambda$ expected events. The 
number of signal events is extracted by minimising the log-likelihood as a function of the signal yield. Using this signal yield, the expected statistical significance and corresponding uncertainty on $\sigma_{(W H, Z H, t \bar{t} H)} \times B R_{H \rightarrow \tau \tau}$ are calculated for a range of input Higgs masses for a given integrated luminosity per experiment, assuming a $10 \%$ systematic uncertainty on the background yield (shown in Figure 1).
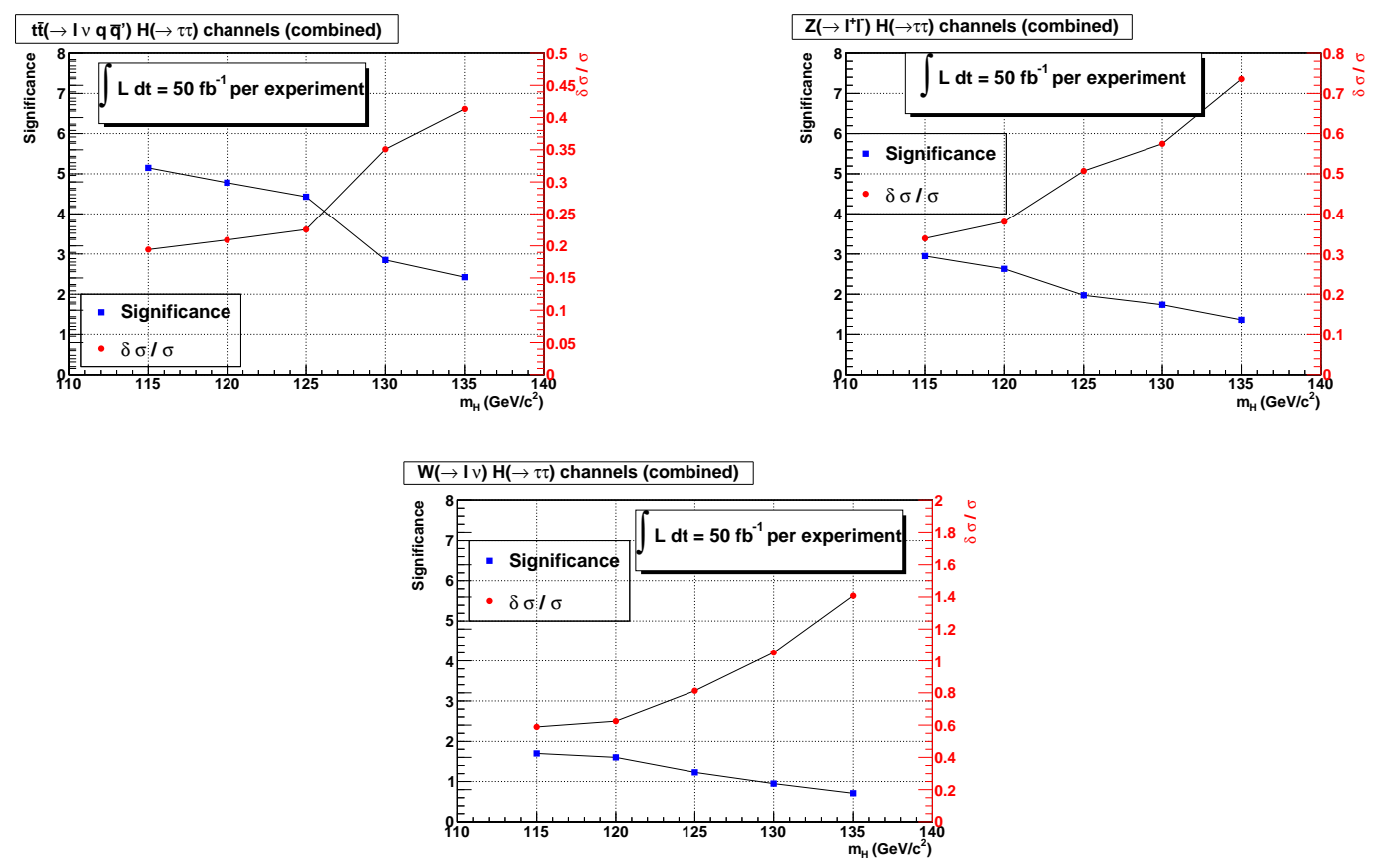

Figure 1: Expected signal significances.

\section{Conclusion}

The prospects for measuring the Higgs boson couplings at the LHC are good in several previously unexplored Higgs production and decay channels. We plan to further refine the selection criteria to improve the expected significance.

\section{References}

[1] L. Evans and P. Bryant, J. Instrum. 3, S08001 (2008).

[2] A. Djouadi, Phys. Rep. 457, 1 (2008), and references therein.

[3] J. M. Butterworth, A. R. Davison, M. Rubin, and G. P. Salam, Phys. Rev. Lett. 100, 242001 (2008).

[4] D. Zeppenfeld, R. Kinnunen, A. Nikitenko, and E. Richter-Was, Phys. Rev. D 62, 013309 (2000).

[5] T. Gleisberg et al., J. High Energy Phys. 0902, 007 (2009). We use version 1.2.1.

[6] M. Bähr et al., Eur. Phys. J. C 58, 639 (2008).

[7] S. Ovyn, X. Rouby, and V. Lemaitre, arXiv:hep-ph/0903.2225v3 (2010).

[8] G. Aad et al. (ATLAS Collaboration), arXiv:0901.0512v4 (2009). 